Pacific

Journal of

Mathematics

FINITE NONSOLVABLE GROUPS WITH MANY DISTINCT CHARACTER DEGREES

Hung P. TONG-VIET 


\title{
FINITE NONSOLVABLE GROUPS WITH MANY DISTINCT CHARACTER DEGREES
}

\author{
Hung P. TONG-VIET
}

\begin{abstract}
Let $G$ be a finite group and let $\operatorname{Irr}(G)$ denote the set of all complex irreducible characters of $G$. Let $\operatorname{cd}(G)$ be the set of all character degrees of $G$. For a degree $d \in \operatorname{cd}(G)$, the multiplicity of $d$ in $G$, denoted by $m_{G}(d)$, is the number of irreducible characters of $G$ having degree $d$. A finite group $G$ is said to be a $T_{k}$-group for some integer $k \geq 1$ if there exists a nontrivial degree $d_{0} \in \operatorname{cd}(G)$ such that $m_{G}\left(d_{0}\right)=k$ and that for every $d \in \operatorname{cd}(G)-\left\{1, d_{0}\right\}$, the multiplicity of $d$ in $G$ is trivial, that is, $m_{G}(d)=1$. In this paper, we show that if $G$ is a nonsolvable $T_{k}$-group for some integer $k \geq 1$, then $k=2$ and $G \cong \mathrm{PSL}_{2}$ (5) or $\mathrm{PSL}_{2}(7)$.
\end{abstract}

\section{Introduction}

Let $G$ be a finite group and let $\operatorname{Irr}(G)=\left\{\chi_{1}, \chi_{2}, \cdots, \chi_{s}\right\}$ be the set of all complex irreducible characters of $G$. Let $\operatorname{cd}(G)=\left\{d_{0}, d_{1}, \cdots, d_{t}\right\}, 1=d_{0}<d_{1}<\cdots<d_{t}$, be the set of all character degrees of $G$. For an integer $d \geq 1$, the multiplicity of $d$ in $G$, denoted by $m_{G}(d)$, is the number of irreducible characters of $G$ having degree $d$; i.e., $m_{G}(d)=|\{\chi \in \operatorname{Irr}(G) \mid \chi(1)=d\}|$. Let $n_{i}=\chi_{i}(1)$ for $1 \leq i \leq s$. We call $\operatorname{mp}(G)=$ $\left(m_{G}\left(d_{0}\right), m_{G}\left(d_{1}\right), \cdots, m_{G}\left(d_{t}\right)\right)$ the multiplicity pattern and $\left(n_{1}, n_{2}, \ldots, n_{s}\right)$ the degree pattern of $G$. Let $\mathbb{C} G$ be the complex group algebra of $G$. We know that $\mathbb{C} G=\bigoplus_{i=1}^{s} \mathrm{M}_{n_{i}}(\mathbb{C})$ and thus knowing the degree pattern of $G$ is equivalent to knowing the structure of the complex group algebra of $G$, or, equivalently, the first column of the ordinary character table of $G$. One of the main questions in character theory of finite groups is Problem 1 in [Brauer 1963], asking for the possible degree patterns of finite groups. It was proved in [Moretó 2007; Craven 2008] that the order of a finite group is bounded in terms of the largest multiplicity of its character degree. This gives a new restriction on the degree patterns of finite groups.

Motivated by this result, we want to explore the relations between the multiplicities of character degrees of finite groups and the structure of the groups.

This research is supported by a Startup Research Fund from the College of Agriculture, Engineering and Science, the University of KwaZulu-Natal.

MSC2010: primary 20C15; secondary 20C33, $20 \mathrm{D} 05$.

Keywords: multiplicity, character degrees, nonsolvable groups. 
This problem has been the subject of much literature; we mention in particular [Berkovich 1996; Berkovich and Kazarin 1996; Berkovich et al. 1992; Dolfi et al. 2013; Seitz 1968]. In this last reference, G. Seitz classified all finite groups which have exactly one nonlinear irreducible representation. This result was generalized in [Berkovich et al. 1992], where the authors classified all finite groups $G$ in which the multiplicity of every nonlinear irreducible character degree $G$ is trivial. Finite groups in which only two nonlinear irreducible characters have equal degrees have been classified in [Berkovich 1996; Berkovich and Kazarin 1996].

To generalize these results, we consider the following definition. A finite group $G$ is called a $T_{k}$-group for some integer $k \geq 1$ if there exists a nontrivial degree $d_{0} \in \operatorname{cd}(G)$ such that $m_{G}\left(d_{0}\right)=k$ and that for every nontrivial degree $d \in \operatorname{cd}(G)$ different from $d_{0}$, we have $m_{G}(d)=1$. Obviously, the finite groups studied in [Berkovich et al. 1992; Seitz 1968] and [Berkovich 1996; Berkovich and Kazarin 1996] are exactly $T_{1}$-groups and $T_{2}$-groups, respectively. In this paper, we generalize the results in [Berkovich and Kazarin 1996] as follows:

Theorem A. Let $G$ be a finite nonsolvable group. If $G$ is a $T_{k}$-group for some integer $k \geq 1$, then $G \cong \mathrm{PSL}_{2}(5)$ or $\mathrm{PSL}_{2}(7)$ and $k=2$.

From [Conway et al. 1985] we know that the multiplicity patterns of $\operatorname{PSL}_{2}(q)$ for $q \in\{5,7\}$ are $(1,2,1,1)$ and $(1,2,1,1,1)$, respectively. Suppose that $G$ is a finite group such that $\operatorname{mp}(G)=\mathrm{mp}\left(\mathrm{PSL}_{2}(q)\right)$ with $q \in\{5,7\}$. Since the first entry of $\operatorname{mp}(G)$ is $\left|G: G^{\prime}\right|=1$, we see that $G$ is perfect and hence a nonsolvable $T_{2}$-group. Applying Theorem A, we deduce that $G \cong \mathrm{PSL}_{2}$ (5) or $\mathrm{PSL}_{2}$ (7). By comparing the number of distinct character degrees, we deduce that $G \cong \operatorname{PSL}_{2}(q)$. It follows that $\mathrm{PSL}_{2}(5)$ and $\mathrm{PSL}_{2}(7)$ are uniquely determined by the multiplicity patterns.

In [Tong-Viet 2013] we conjectured that every nonabelian simple group is uniquely determined by its multiplicity pattern, and showed that this conjecture holds for every nonabelian simple group with at most 7 distinct character degrees. If true, this conjecture generalizes a result in [Tong-Viet 2012] saying that all nonabelian simple groups are uniquely determined by the structure of their complex group algebras. This latter result is related to Problem 2 in [Brauer 1963], which asks: What does $\mathbb{C} G$ know about $G$ ? This is also an important question in character theory and has been studied extensively (see the references in [Tong-Viet 2012]).

Notice that if the degree pattern of a finite group $G$ is given, then both $\operatorname{cd}(G)$ and $\operatorname{mp}(G)$ are known. Thus, apart from being a direct generalization of the results obtained in [Berkovich and Kazarin 1996], Theorem A could be used to study questions raised in [Tong-Viet 2013]. For finite solvable groups, if $G$ is a finite $T_{k}$-group of odd order, then $|\operatorname{cd}(G)| \leq 2$ since $G$ has only one real irreducible character (the trivial character) and thus every nontrivial character degree of $G$ has multiplicity at least 2 . On the other hand, every finite group with exactly two 
distinct character degrees is a solvable $T_{k}$-group for some integer $k$ (see [Isaacs 1976, Corollary 12.6]) and a compete classification of such finite groups is yet to be found. This together with the fact that there is no explicit upper bound for $k$ makes the classification of solvable $T_{k}$-groups quite complicated even for 2-groups.

Throughout this paper, all groups are finite and all characters are complex characters. Let $G$ be a group. If $N \unlhd G$ and $\theta \in \operatorname{Irr}(N)$, the inertia group of $\theta$ in $G$ is denoted by $I_{G}(\theta)$. We write $\operatorname{Irr}(G \mid \theta)$ for the set of all irreducible constituents of $\theta^{G}$. The order of an element $x \in G$ is denoted by $|x|$. Denote by $\Phi_{k}:=\Phi_{k}(q)$ the value of the $k$-th cyclotomic polynomial evaluated at $q$. Other notation is standard.

\section{Preliminaries}

Lemma 2.1. Let $G$ be a group and let $N \unlhd G$ be such that $G / N$ is cyclic of order $d \geq 2$. Assume that $G$ has a nontrivial degree a with multiplicity $m$. Suppose that $a>d$ and $m / d \geq 2$. Then $N$ has a nontrivial degree $b$ with multiplicity at least 2 and $a / d \leq b \leq a$.

Proof. Assume first that $\chi_{N}$ is not irreducible for some $\chi \in \operatorname{Irr}(G)$ with $\chi(1)=a$. Let $\theta \in \operatorname{Irr}(N)$ be an irreducible constituent of $\chi_{N}$. As $G / N$ is cyclic, we deduce from [Isaacs 1976, Corollary 11.22] that $\theta$ is not $G$-invariant. Let $I=I_{G}(\theta)$ and $t:=|G: I|$. Then $t \geq 2$. By Clifford's theorem [Isaacs 1976, Theorem 6.2] and the corollary just cited, we deduce that $\chi_{N}=\sum_{i=1}^{t} \theta_{i}$, where $\theta_{i} \in \operatorname{Irr}(N)$ are distinct conjugates of $\theta$. Hence $N$ has a nontrivial degree $a / t$ with multiplicity at least $t \geq 2$. Since $t$ divides $|G / N|=d$, we deduce that $a / d \leq a / t \leq a$. Assume now that $\chi_{N} \in \operatorname{Irr}(N)$ for every $\chi \in \operatorname{Irr}(G)$ with $\chi(1)=a$. It follows that $N$ has a nontrivial degree $a$ with multiplicity at least $m / d \geq 2$ as each irreducible character $\chi_{N} \in \operatorname{Irr}(N)$ has exactly $d$ extensions in $G$. Therefore, in both cases $N$ has a nontrivial degree $b$ with $1<a / d \leq b \leq a$ with multiplicity at least 2 . The proof is now complete.

We note that when $d$ in the previous lemma is a prime, then $b \in\{a, a / d\}$. As an application of this lemma, we obtain:

Corollary 2.2. Let $G$ be a group and let $N \unlhd G$ be such that $G / N$ is cyclic of order $d \geq 2$. Assume that $G$ has two nontrivial degrees $a_{1}, a_{2}$, with multiplicities $m_{1}, m_{2}$. Suppose that $a_{2} / d>a_{1}>d$ and $m_{i} \geq 2 d$ for $i=1,2$. Then $N$ is not a $T_{k}$-group for any integer $k \geq 1$.

Proof. By Lemma 2.1, $N$ has two nontrivial character degrees $d_{1}, d_{2}$, each with multiplicity at least 2 , such that $a_{i} / d \leq d_{i} \leq a_{i}$, for $i=1,2$. Now we have $d_{2} \geq a_{2} / d>a_{1} \geq d_{1} \geq a_{1} / d>1$ by the hypothesis. Hence $d_{1}$ and $d_{2}$ are character degrees of $N$ and both degrees have nontrivial multiplicity, so $N$ is not a $T_{k}$-group for any integer $k \geq 1$.

The next result is well known. See [Carter 1985, §§ 13.8, 13.9] for the notion of 
symbols and the classification of unipotent characters of finite groups of Lie type.

Lemma 2.3. Let $S$ be a nonabelian simple group.

(1) If $S$ is a sporadic simple group, the Tits group or an alternating group of degree at least 7 , then $S$ has two nontrivial irreducible characters, with distinct degrees and both extendible to $\operatorname{Aut}(S)$.

(2) If $S$ is a simple group of Lie type in characteristic $p$ and $S \neq{ }^{2} \mathrm{~F}_{4}(2)$, then the Steinberg character of $S$, denoted by $\mathrm{St}_{S}$, of degree $|S|_{p}$, is extendible to $\operatorname{Aut}(S)$. Furthermore, if $S ¥ \mathrm{PSL}_{2}\left(3^{f}\right)$, then $S$ possesses an irreducible character $\theta$ such that $\theta(1) \neq|S|_{p}$ and $\theta$ also extends to $\operatorname{Aut}(S)$.

Proof. The first statement follows from [Bianchi et al. 2007, Theorems 3 and 4]. For (2), the existence and extendability of the Steinberg character of $S$ is well known. Now assume that $S \varsubsetneqq \operatorname{PSL}_{2}(q)$ with $q=p^{f}$. We can choose $\theta$ to be any unipotent character of $S$ which is not one of the exceptions in [Malle 2008, Theorem 2.5] and not the Steinberg character of $S$, then $\theta$ is extendible to $\operatorname{Aut}(S)$ [Carter 1985, $\S 13.8,13.9]$. Finally, assume that $S \cong \operatorname{PSL}_{2}(q)$ with $q=p^{f}$ and $p \neq 3$. Then $S$ has an irreducible character $\theta$ of degree $q+\delta$, where $q \equiv \delta(\bmod 3)$ and $\delta \in\{ \pm 1\}$ such that $\theta$ extends to $\operatorname{Aut}(S)$. Notice that this irreducible character of $S$ corresponds to a semisimple element of order 3 in the dual group $\mathrm{SL}_{2}(q)$.

Lemma 2.4 [Zsigmondy 1892]. Let $q \geq 2$ and $n \geq 3$ be integers such that $(n, q) \neq$ $(6,2)$. Then $q^{n}-1$ has a prime factor $\ell$ such that $\ell \equiv 1(\bmod n)$ and $\ell$ does not divide $q^{m}-1$ for any $m<n$.

Such an $\ell$ is called a primitive prime divisor and is denoted by $\ell_{n}(q)$.

The orders of two maximal tori and the corresponding primitive prime divisors of the finite classical groups are given in Table 1, taken from [Malle 1999, Table 3.5]. Table 2 lists the degrees of some unipotent characters of the simple exceptional groups of Lie type. This can be found in [Carter 1985, §13.9].

\section{Simple $T_{k}$-groups}

The main purpose of this section is to classify all simple $T_{k}$-groups. As we will see shortly, there are only two simple $T_{k}$-groups and they are exactly the simple $T_{2}$-groups. Let $\mathscr{L}$ be the set consisting of the following simple groups:

$$
\begin{gathered}
\operatorname{PSL}_{2}(q), \operatorname{PSL}_{3}(q), \operatorname{PSU}_{3}(q), \operatorname{PSp}_{4}(q), \\
\operatorname{PSL}_{6}(2), \operatorname{PSL}_{7}(2), \operatorname{PSU}_{4}(2), \operatorname{PSp}_{6}(2), \operatorname{PSp}_{8}(2), \mathrm{P}_{8}^{ \pm}(2)
\end{gathered}
$$

and

$$
\begin{gathered}
\mathrm{PSL}_{4}(2), \mathrm{PSU}_{4}(3), \mathrm{PSU}_{5}(2), \mathrm{PSp}_{6}(3), \\
\Omega_{7}(3), \mathrm{PSp}_{8}(3), \Omega_{9}(3), \mathrm{P} \Omega_{8}^{ \pm}(3), \mathrm{P} \Omega_{10}^{+}(2), \mathrm{P} \Omega_{10}^{-}(3) .
\end{gathered}
$$




\begin{tabular}{cllll}
\hline$G=G(q)$ & \multicolumn{1}{c}{$\left|T_{1}\right|$} & $\left|T_{2}\right|$ & \multicolumn{1}{c}{$\ell_{1}$} & $\ell_{2}$ \\
\hline $\mathrm{A}_{n}$ & $\left(q^{n+1}-1\right) /(q-1)$ & $q^{n}-1$ & $\ell_{n+1}(q)$ & $\ell_{n}(q)$ \\
${ }^{2} \mathrm{~A}_{n},(n \equiv 0(4))$ & $\left(q^{n+1}+1\right) /(q+1)$ & $q^{n}-1$ & $\ell_{2 n+2}(q)$ & $\ell_{n}(q)$ \\
${ }^{2} \mathrm{~A}_{n},(n \equiv 1(4))$ & $\left(q^{n+1}-1\right) /(q+1)$ & $q^{n}+1$ & $\ell_{(n+1) / 2}(q)$ & $\ell_{2 n}(q)$ \\
${ }^{2} \mathrm{~A}_{n},(n \equiv 2(4))$ & $\left(q^{n+1}+1\right) /(q+1)$ & $q^{n}-1$ & $\ell_{2 n+2}(q)$ & $\ell_{n / 2}(q)$ \\
${ }^{2} \mathrm{~A}_{n},(n \equiv 3(4))$ & $\left(q^{n+1}-1\right) /(q+1)$ & $q^{n}+1$ & $\ell_{n+1}(q)$ & $\ell_{2 n}(q)$ \\
$\mathrm{B}_{n}, \mathrm{C}_{n}(n \geq 3$ odd $)$ & $q^{n}+1$ & $q^{n}-1$ & $\ell_{2 n}(q)$ & $\ell_{n}(q)$ \\
$\mathrm{B}_{n}, \mathrm{C}_{n}(n \geq 2$ even $)$ & $q^{n}+1$ & $\left(q^{n-1}+1\right)(q+1)$ & $\ell_{2 n}(q)$ & $\ell_{2 n-2}(q)$ \\
$\mathrm{D}_{n},(n \geq 5$ odd $)$ & $\left(q^{n-1}+1\right)(q+1)$ & $q^{n}-1$ & $\ell_{2 n-2}(q)$ & $\ell_{n}(q)$ \\
$\mathrm{D}_{n},(n \geq 4$ even $)$ & $\left(q^{n-1}+1\right)(q+1)$ & $\left(q^{n-1}-1\right)(q-1)$ & $\ell_{2 n-2}(q)$ & $\ell_{n-1}(q)$ \\
${ }^{2} \mathrm{D}_{n}$ & $q^{n}+1$ & $\left(q^{n-1}+1\right)(q-1)$ & $\ell_{2 n}(q)$ & $\ell_{2 n-2}(q)$ \\
\hline
\end{tabular}

Table 1. Two tori for classical groups.

The following result will be needed when dealing with simple classical groups of Lie type. We refer to [Dolfi et al. 2013, §4.3] and [Larsen et al. 2013, Theorem 4.7] for some related results.

Lemma 3.1. Let $G$ be a simply connected simple algebraic group of classical type and let $F$ be a suitable Frobenius map such that $S \cong \mathscr{G}^{F} / \mathrm{Z}\left(\varphi^{F}\right)$ is a simple classical group of Lie type defined over a finite field of size $q$ with $S \notin \mathscr{L}$. Let the pair $\left(\varphi^{*}, F^{*}\right)$ be dual to $(\mathscr{G}, F)$ and let $G=\left(\mathscr{G}^{*}\right)^{F^{*}}$. For $i=1,2$, let $T_{i}$ be the maximal tori of $G$ with order given in Table 1. Then for each $i$, there exist two regular semisimple elements $s_{i}, t_{i} \in T_{i}$ such that $s_{i}, t_{i} \in T_{i} \cap G^{\prime}$ and that $s_{i}$ and $t_{i}$ are not $G$-conjugate.

Proof. Since $G$ is of simply connected type, the dual group $G^{*}$ is of adjoint type and thus by using the identifications with classical groups in [Carter 1985, page 40], $G / S$ is either a cyclic or an elementary abelian group of order 4 . In all cases, $G / S$ is abelian and so $G^{\prime}=S$. For each $i=1,2$, let $T_{i}^{\prime}=T_{i} \cap G^{\prime}$. Since $G^{\prime} \unlhd T_{i} G^{\prime} \leq G$, we obtain that $\left|T_{i}^{\prime}\right|=\left|T_{i} \cap G^{\prime}\right| \geq\left|T_{i}\right| / d$ with $d:=\left|G: G^{\prime}\right|$. Since $S \notin \mathscr{L}$, the primitive prime divisors $\ell_{1}$ and $\ell_{2}$ in Table 1 both exist.

Claim 1. For $i=1,2$, every element $s_{i} \in T_{i}$ of order $\ell_{i}$ is a regular semisimple element and $s_{i} \in T_{i} \cap G^{\prime}=T_{i}^{\prime}$.

Observe that the two maximal tori of $G$ with order given in Table 1 have the properties that they are uniquely determined up to conjugation by their orders. Furthermore, for each $i=1,2$, the conjugacy class of maximal tori containing $T_{i}$ is the only class of maximal tori whose order is divisible by $\ell_{i}$. Also, the Sylow $\ell_{i}$-subgroups of $G$ are cyclic. Let $s_{i} \in T_{i}$ be a semisimple element of order $\ell_{i}$. As in the proof of [Malle 2010, Proposition 2.4], if $\mathrm{C}_{G}\left(s_{i}\right)$ is not a torus, then its semisimple rank is at least 1 , and thus it contains two maximal tori of different 
orders. Both of these tori must have orders divisible by $\ell_{i}$, which is impossible. Hence we obtain that $\mathrm{C}_{G}\left(s_{i}\right)=T_{i}$. Since $\operatorname{gcd}\left(\ell_{i},\left|G: G^{\prime}\right|\right)=1$, we deduce that $s_{i} \in G^{\prime}$ and so $s_{i} \in T_{i}^{\prime}=T_{i} \cap G^{\prime}$.

Claim 2. For every $x \in T_{i}$, if $|x|$ is divisible by $\ell_{i}$, then $x$ is a regular semisimple element.

Assume that $x \in T_{i}$ such that $|x|=m \ell_{i}$ where $m \geq 1$ is an integer. Let $s=x^{m} \in T_{i}$. Then $|s|=\ell_{i}$ and so by Claim 1 , we know that $\mathrm{C}_{G}(s)=T_{i}$, hence $T_{i} \leq \mathrm{C}_{G}(x) \leq$ $\mathrm{C}_{G}\left(x^{m}\right)=\mathrm{C}_{G}(s)=T_{i}$. So, $\mathrm{C}_{G}(x)=T_{i}$ and $x$ is a regular semisimple element.

Claim 3. If $T_{i}^{\prime}=T_{i} \cap G^{\prime}$ has no element whose order is a proper multiple of $\ell_{i}$, then $T_{i}^{\prime}$ contains two distinct $G$-conjugacy classes of regular semisimple elements of order $\ell_{i}$.

For $i=1,2$, let $T_{i}^{\prime \prime}$ be a cyclic subgroup of $T_{i}^{\prime}$ whose order is divisible by $\ell_{i}$. By our assumption, we must have that $\left|T_{i}^{\prime \prime}\right|=\ell_{i}$ and so $T_{i}^{\prime \prime}=\left\langle s_{i}\right\rangle$, where $s_{i}$ is an element of order $\ell_{i}$. As $s_{i}$ is regular semisimple by Claim 1, we deduce that $\mathrm{N}_{G}\left(\left\langle s_{i}\right\rangle\right) \leq$ $\mathrm{N}_{G}\left(T_{i}\right)$ and so as the Sylow $\ell_{i}$-subgroup of $T_{i}$ is cyclic and $\left|s_{i}\right|=\ell_{i}$, we obtain that $\mathrm{N}_{G}\left(\left\langle s_{i}\right\rangle\right)=\mathrm{N}_{G}\left(T_{i}\right)$. Since $\mathrm{C}_{G}\left(s_{i}\right)=T_{i}=\mathrm{C}_{G}\left(T_{i}\right)$ and $\left|\mathrm{N}_{G}\left(T_{i}\right) / T_{i}\right| \leq m(S)$, we deduce that $\left|\mathrm{N}_{G}\left(\left\langle s_{i}\right\rangle\right) / \mathrm{C}_{G}\left(s_{i}\right)\right| \leq m(S)$, where $m(S)$ is the dimension of the natural module for $S=G^{\prime}$ over $\mathbb{F}_{q}$. (Notice that the fact $\left|\mathrm{N}_{G}\left(T_{i}\right) / T_{i}\right| \leq m(S)$ can be deduced from [Babai et al. 2009, Lemma 4.7].) It follows that $s_{i}$ is $G$-conjugate to at most $m(S)$ of its powers and thus $T_{i}^{\prime \prime}=\left\langle s_{i}\right\rangle$ contains at least $\varphi\left(\left|s_{i}\right|\right) / m(S) G$-conjugacy classes of regular semisimple elements of order $\ell_{i}$, where $\varphi$ is the Euler $\varphi$-function. Since $\left|s_{i}\right|=\ell_{i}$, we deduce that $\varphi\left(\left|s_{i}\right|\right) / m(S)=\left(\left|s_{i}\right|-1\right) / m(S) \geq\left(\left|T_{i}^{\prime \prime}\right|-1\right) / m(S)$. We now verify that for each possibility of $S$, we have that $\left(\left|T_{i}^{\prime \prime}\right|-1\right) / m(S) \geq 2$, which implies that $T_{i}^{\prime}$ contains at least two distinct $G$-conjugacy classes of regular semisimple elements of order $\ell_{i}$.

(a) Assume first that $S \cong \operatorname{PSL}_{n}(q)$. Then $m(S)=n$ and $d=\operatorname{gcd}(n, q-1)$. Since $T_{i}$, $i=1,2$, are cyclic, we deduce that both $T_{i}^{\prime}$ are also cyclic of order at least $\left|T_{i}\right| / d$. Hence we can choose $T_{i}^{\prime \prime}=T_{i}^{\prime}$ for $i=1,2$. Then $\left|T_{1}^{\prime \prime}\right| \geq\left(q^{n}-1\right) /(d(q-1))$ and $\left|T_{2}^{\prime \prime}\right| \geq\left(q^{n-1}-1\right) / d$. As $S \notin \mathscr{L}$, it is routine to check that $q^{n-1}-1 \geq(2 n+1)(q-1)$ and so since $q-1 \geq d=\operatorname{gcd}(n, q-1)$, we obtain that $q^{n-1}-1 \geq(2 n+1) d$ or equivalently $\left(\left|T_{2}^{\prime \prime}\right|-1\right) / n \geq 2$. Similarly, we can check that $\left(\left|T_{1}^{\prime \prime}\right|-1\right) / n \geq 2$.

(b) Assume that $S \cong \operatorname{PSU}_{n}(q)$ and $n \geq 5$ is odd. We have that $m(S)=n$ and $d=\operatorname{gcd}(n, q+1)$. As in the previous case, we see that both $T_{i}$ are cyclic and so are $T_{i}^{\prime}$, hence we can choose $T_{i}^{\prime \prime}=T_{i}^{\prime}$. Then $\left|T_{1}^{\prime \prime}\right| \geq\left(q^{n}+1\right) /(d(q+1))$ and $\left|T_{2}^{\prime \prime}\right| \geq\left(q^{n-1}-1\right) / d$. Since $q^{n-1}-1>\left(q^{n}+1\right) /(q+1)$ and $d=\operatorname{gcd}(n, q+1) \leq q+1$, it suffices to show that $\left(q^{n}+1\right) \geq(2 n+1)(q+1)^{2}$ with $n \geq 5$ odd. Since $S \notin \mathscr{L}$, we can check that the previous inequality holds so that $\left(\left|T_{i}^{\prime \prime}\right|-1\right) / m(S) \geq 2$ for $i=1,2$, as required. 
(c) Assume that $S \cong \operatorname{PSU}_{n}(q)$ and $n \geq 4$ is even. Arguing as in the case $n$ is odd, we have that $\left|T_{1}^{\prime \prime}\right| \geq\left(q^{n}-1\right) /(d(q+1))$ and $\left|T_{2}^{\prime \prime}\right| \geq\left(q^{n-1}+1\right) / d$. Since $q^{n-1}+1>$ $\left(q^{n}-1\right) /(q+1)$ and $d \leq q+1$, it suffices to show that $q^{n}-1 \geq(2 n+1)(q+1)^{2}$ where $n \geq 4$ is even. As $S \notin \mathscr{L}$, we can check that the previous inequality holds so that $\left(q^{n}-1\right) /\left(n(q+1)^{2}\right) \geq 2$ and thus $\left(\left|T_{i}^{\prime \prime}\right|-1\right) / m(S) \geq 2$ for $i=1,2$, as required.

(d) Assume that $S \cong \operatorname{PSp}_{2 n}(q)$ or $\Omega_{2 n+1}(q)$ and $n \geq 3$ is odd. We have that $m(S) \leq 2 n+1$ and $d=\operatorname{gcd}(2, q-1)$. In this case both $T_{i}$ are cyclic, so we can choose $T_{i}^{\prime \prime}=T_{i}^{\prime}$ and hence $\left|T_{1}^{\prime \prime}\right| \geq\left(q^{n}+1\right) / d$ and $\left|T_{2}^{\prime \prime}\right| \geq\left(q^{n}-1\right) / d$. Since $q^{n}+1>q^{n}-1$, it suffices to show that $q^{n}-1 \geq(4 n+3) d$, where $n \geq 3$ is odd and $d=\operatorname{gcd}(2, q-1)$. Since $S \notin \mathscr{L}$, we can check that the latter inequality holds, so for $i=1,2$, we obtain that $\left(\left|T_{i}^{\prime \prime}\right|-1\right) / m(S) \geq 2$.

(e) Assume that $S \cong \operatorname{PSp}_{2 n}(q)$ or $\Omega_{2 n+1}(q)$ and $n \geq 4$ is even. We can choose $\left|T_{1}^{\prime \prime}\right|=\left|T_{1}^{\prime}\right| \geq\left(q^{n}+1\right) / d$ and $\left|T_{2}^{\prime \prime}\right| \geq\left(q^{n-1}+1\right) / d$. Since $q^{n}+1>q^{n-1}+1$, it suffices to show that $q^{n-1}+1 \geq(4 n+3) d$, where $n \geq 4$ is even and $d=\operatorname{gcd}(2, q-1)$. Since $S \notin \mathscr{L}$, we can check that the latter inequality holds, so for $i=1,2$, we have that $\left(\left|T_{i}^{\prime \prime}\right|-1\right) / m(S) \geq 2$.

(f) Assume that $S \cong P \Omega_{2 n}^{+}(q)$ where $n \geq 5$ is odd. Then $m(S)=2 n$ and $d=$ $\operatorname{gcd}\left(4, q^{n}-1\right)$. We have $\left|T_{1}^{\prime \prime}\right| \geq\left(q^{n-1}+1\right) / d$ and $\left|T_{2}^{\prime \prime}\right| \geq\left(q^{n}-1\right) / d$. Since $q^{n}-1>q^{n-1}+1$, it suffices to show that $q^{n-1}+1 \geq(4 n+1) d$, where $n \geq 5$ is odd. Since $S \notin \mathscr{L}$, we can check that the latter inequality holds, so for $i=1,2$, we obtain that $\left(\left|T_{i}^{\prime \prime}\right|-1\right) / m(S) \geq 2$.

(g) Assume that $S \cong P \Omega_{2 n}^{+}(q)$ where $n \geq 4$ is even. Then $\left|T_{1}^{\prime \prime}\right| \geq\left(q^{n-1}+1\right) / d$ and $\left|T_{2}^{\prime \prime}\right| \geq\left(q^{n-1}-1\right) / d$. Since $q^{n-1}+1>q^{n-1}-1$, it suffices to show that $q^{n-1}-1 \geq(4 n+1) d$, where $n \geq 4$ is even. Since $S \notin \mathscr{L}$, we can check that the latter inequality holds, so for $i=1,2,\left(\left|T_{i}^{\prime \prime}\right|-1\right) / m(S) \geq 2$.

(h) Assume that $S \cong P \Omega_{2 n}^{-}(q)$ where $n \geq 4$. Then $\left|T_{1}^{\prime \prime}\right| \geq\left(q^{n}+1\right) / d$ and $\left|T_{2}^{\prime \prime}\right| \geq$ $\left(q^{n-1}+1\right) / d$. Since $q^{n}+1>q^{n-1}+1$, it suffices to show that $q^{n-1}+1 \geq(4 n+1) d$, where $n \geq 4$. Since $S \notin \mathscr{L}$, we can check that the latter inequality holds, so for $i=1,2,\left(\left|T_{i}^{\prime \prime}\right|-1\right) / m(S) \geq 2$ as wanted. This completes the proof of Claim 3 .

Finally, by Claim 1, to finish the proof of the lemma, we only need to find a regular semisimple element $t_{i} \in T_{i}^{\prime}$ such that $t_{i}$ is not $G$-conjugate to $s_{i}$ for $i=1,2$. Now, for each $i$, if $T_{i}^{\prime}$ contains an element whose order is a proper multiple of $\ell_{i}$, then this element is a regular semisimple element by Claim 2 and clearly it is not $G$-conjugate to $s_{i}$ as the orders of these two semisimple elements are distinct. Otherwise, if no such elements exists, then by Claim 3 we can find a regular semisimple element $t_{i} \in T_{i}^{\prime}$ with the same order as that of $s_{i}$ and they are not $G$-conjugate. The proof is now complete. 
We now prove the main result of this section.

Theorem 3.2. Let $S$ be a nonabelian simple group. If $S$ is a $T_{k}$-group for some integer $k \geq 1$, then $k=2$ and $S \cong \mathrm{PSL}_{2}$ (5) or $\mathrm{PSL}_{2}$ (7).

Proof. Using the classification of finite simple groups, we consider several cases:

(1) $S$ is a sporadic simple group or the Tits group. It is routine to check using [Conway et al. 1985] that $S$ has at least two nontrivial distinct degrees, each with multiplicity at least 2 . Hence $S$ is not a $T_{k}$-group for any integer $k \geq 1$.

(2) $S \cong \mathrm{A}_{n}$ with $n \geq 5$. If $n=5$, then $\operatorname{cd}\left(\mathrm{A}_{5}\right)=\{1,3,4,5\}$ and every degree of $A_{5}$ has multiplicity 1 , except for the degree 3 with multiplicity 2 . Hence $A_{5}$ is a $T_{2}$-group. Now assume that $n \geq 6$. For $6 \leq n \leq 13$, we can check that $\mathrm{A}_{n}$ is not a $T_{k}$-group by using [Conway et al. 1985]. Thus we can assume that $n \geq 14$. Let $\lambda$ be a self-conjugate partition of $n$ and denote by $\chi^{\lambda}$ the irreducible character of $\mathrm{S}_{n}$ labeled by $\lambda$. Then $\chi^{\lambda}$ when restricted to $\mathrm{A}_{n}$ will split into the sum of two irreducible characters having the same degree $\chi^{\lambda}(1) / 2$. Thus $\chi^{\lambda}(1) / 2 \in \operatorname{cd}\left(A_{n}\right)$ has multiplicity at least two. Therefore, in order to show that $\mathrm{A}_{n}$ is not a $T_{k}$-group for any $k \geq 1$, it suffices to find two distinct self-conjugate partitions $\lambda_{i}, i=1,2$, of $n$ such that $\chi^{\lambda_{i}}(1) / 2, i=1,2$, are distinct and nontrivial.

Assume first that $n \geq 15$ is odd. We can write $n=2 k+9=2(k+4)+1$. Then $\lambda_{1}=\left(k+5,1^{k+4}\right)$ and $\lambda_{2}=\left(k+3,3^{2}, 1^{k}\right)$ are two distinct self-conjugate partitions of $n$. Assume next that $n \geq 14$ is even. Write $n=2 k+8$. Then $\lambda_{1}=\left(k+4,2,1^{k+2}\right)$ and $\lambda_{2}=\left(k+3,3^{2}, 1^{k}\right)$ are two distinct self-conjugate partitions of $n$. Using Hook formula, we can easily check that $\chi^{\lambda_{i}}(1) / 2$ are distinct and nontrivial for $i=1,2$. Thus $\mathrm{A}_{n}$ is not a $T_{k}$-group for $n \geq 14$.

(3) $S$ is a simple exceptional group of Lie type in characteristic $p$.

Assume first that $S \cong{ }^{2} \mathrm{~B}_{2}\left(q^{2}\right)$, where $q^{2}=2^{2 m+1}$ and $m \geq 1$. By [Suzuki 1962], $S$ has irreducible characters of degree $\sqrt{2} q\left(q^{2}-1\right) / 2$ and $q^{4}+1$, with multiplicity 2 and $\left(q^{2}-2\right) / 2$, respectively. Hence $S$ is not a $T_{k}$-group for any $k \geq 1$.

Assume next that $S \cong{ }^{3} \mathrm{D}_{4}(q)$. If $q=2$, then ${ }^{3} \mathrm{D}_{4}(2)$ is not a $T_{k}$-group for any integer $k \geq 1$ by using [Conway et al. 1985]. Hence we can assume that $q \geq 3$. By [Deriziotis and Michler 1987, Table 4.4], $S$ has degrees given by

$$
\left(q^{3}+\delta\right)\left(q^{2}-\delta q+1\right)\left(q^{4}-q^{2}+1\right)
$$

with multiplicity $\frac{1}{2} q(q+\delta)$, where $\delta= \pm 1$. Since $q \geq 3$, we deduce that these two degrees are distinct and nontrivial and $q(q+1) / 2 \geq q(q-1) / 2 \geq 3$, so $S$ is not a $T_{k}$-group.

Assume that $S \cong \mathrm{E}_{6}(q)$ and let $G=\mathrm{E}_{6}(q)_{\text {ad. }}$. Let $d=|G: S|=\operatorname{gcd}(3, q-1)$. By [Lübeck 2007], $G$ has an irreducible character $\chi$ of degree

$$
\chi(1)=\frac{1}{2} q^{3} \Phi_{1}^{2} \Phi_{3}^{2} \Phi_{4} \Phi_{5} \Phi_{6}^{2} \Phi_{8} \Phi_{9} \Phi_{12}, \quad \text { with } m_{G}(\chi(1)) \geq q(q-1) .
$$




\begin{tabular}{clr}
\hline$S=S(q)$ & Symbol & Degree \\
\hline${ }^{2} \mathrm{G}_{2}\left(q^{2}\right)$ & cuspidal & $\frac{1}{\sqrt{3}} q \Phi_{1} \Phi_{2} \Phi_{4}$ \\
& cuspidal & $\frac{1}{2 \sqrt{3}} q \Phi_{1} \Phi_{2} \Phi_{12}^{\prime}$ \\
${ }^{2} \mathrm{~F}_{4}\left(q^{2}\right)$ & $\left({ }^{2} \mathrm{~B}_{2}[a], 1\right),\left({ }^{2} \mathrm{~B}_{2}[b], 1\right)$ & $\frac{1}{\sqrt{2}} q \Phi_{1} \Phi_{2} \Phi_{4}^{2} \Phi_{6}$ \\
& $\left({ }^{2} \mathrm{~B}_{2}[a], \epsilon\right),\left({ }^{2} \mathrm{~B}_{2}[b], \epsilon\right)$ & $\frac{1}{\sqrt{2}} q^{13} \Phi_{1} \Phi_{2} \Phi_{4}^{2} \Phi_{6}$ \\
$\mathrm{G}_{2}(q)$ & $\phi_{1,3}^{\prime}, \phi_{1,3}^{\prime \prime}$ & $\frac{1}{3} q \Phi_{3} \Phi_{6}$ \\
& $\mathrm{G}_{2}[\theta], \mathrm{G}_{2}\left[\theta^{2}\right]$ & $\frac{1}{3} q \Phi_{1}^{2} \Phi_{2}^{2}$ \\
$\mathrm{~F}_{4}(q)$ & $\phi_{8,3}^{\prime}, \phi_{8,3}^{\prime \prime}$ & $q^{3} \Phi_{4}^{2} \Phi_{8} \Phi_{12}$ \\
& $\phi_{8,9}^{\prime}, \phi_{8,9}^{\prime \prime}$ & $q^{9} \Phi_{4}^{2} \Phi_{8} \Phi_{12}$ \\
${ }^{2} \mathrm{E}_{6}(q)$ & ${ }^{2} \mathrm{E}_{6}[\theta],{ }^{2} \mathrm{E}_{6}\left[\theta^{2}\right]$ & $\frac{1}{3} q^{7} \Phi_{1}^{4} \Phi_{2}^{6} \Phi_{4}^{2} \Phi_{8} \Phi_{10}$ \\
$\mathrm{E}_{6}(q)$ & $\mathrm{E}_{6}[\theta], \mathrm{E}_{6}\left[\theta^{2}\right]$ & $\frac{1}{3} q^{7} \Phi_{1}^{6} \Phi_{2}^{4} \Phi_{4}^{2} \Phi_{5} \Phi_{8}$ \\
$\mathrm{E}_{7}(q)$ & $\mathrm{E}_{7}[\xi], \mathrm{E}_{7}[-\xi]$ & $\frac{1}{2} q^{11} \Phi_{1}^{7} \Phi_{3}^{3} \Phi_{4}^{2} \Phi_{5} \Phi_{7} \Phi_{8} \Phi_{9} \Phi_{12}$ \\
& $\mathrm{E}_{6}[\theta], \mathrm{E}_{6}\left[\theta^{2}\right]$ & $\frac{1}{3} q^{7} \Phi_{1}^{6} \Phi_{2}^{6} \Phi_{4}^{2} \Phi_{5} \Phi_{7} \Phi_{8} \Phi_{10} \Phi_{14}$ \\
$\mathrm{E}_{8}(q)$ & $\left(\mathrm{E}_{7}[\xi], 1\right),\left(\mathrm{E}_{7}[-\xi], 1\right)$ & $\frac{1}{2} q^{11} \Phi_{1}^{7} \Phi_{3}^{4} \Phi_{4}^{4} \Phi_{5}^{2} \Phi_{7} \Phi_{8}^{2} \Phi_{9} \Phi_{12}^{2} \Phi_{15} \Phi_{20} \Phi_{24}$ \\
& $\left(\mathrm{E}_{7}[\xi], \epsilon\right),\left(\mathrm{E}_{7}[-\xi], \epsilon\right)$ & $\frac{1}{2} q^{26} \Phi_{1}^{7} \Phi_{3}^{4} \Phi_{4}^{4} \Phi_{5}^{2} \Phi_{7} \Phi_{8}^{2} \Phi_{9} \Phi_{12}^{2} \Phi_{15} \Phi_{20} \Phi_{24}$ \\
\hline
\end{tabular}

Table 2. Some unipotent characters of simple exceptional groups of Lie type.

Obviously, $\chi(1)>d$ and $m_{G}(\chi(1)) \geq 2 d$. By Lemma 2.1, $S$ has a nontrivial degree $b \in\{\chi(1), \chi(1) / d\}$ with nontrivial multiplicity. By Table $2, S$ also has a nontrivial degree $\psi(1)$ with multiplicity at least 2 . Observe that $\psi(1) \notin\{\chi(1), \chi(1) / d\}$. Therefore, $S$ has two distinct nontrivial degrees, each with multiplicity at least 2 , so $S$ is a not a $T_{k}$-group.

The same argument applies to the simple group $S \cong{ }^{2} \mathrm{E}_{6}(q)$ with $q>2$ since $G={ }^{2} \mathrm{E}_{6}(q)_{\text {ad }}$ has a degree

$$
\chi(1)=\frac{1}{2} q^{3} \Phi_{2}^{2} \Phi_{3} \Phi_{4} \Phi_{6}^{3} \Phi_{8} \Phi_{10} \Phi_{12} \Phi_{18}, \quad \text { with } m_{G}(\chi(1)) \geq(q+1)(q-2),
$$

$|G: S|=\operatorname{gcd}(3, q+1)=: d$; and $S$ has a nontrivial degree $\psi(1) \notin\{\chi(1), \chi(1) / d\}$ with multiplicity at least 2 by Table 2 . For the case $q=2$, we can check that ${ }^{2} \mathrm{E}_{6}(2)$ is not a $T_{k}$-group by using [Conway et al. 1985].

Finally, for the remaining simple exceptional groups of Lie type, by Table 2 each simple group $S$ has two distinct nontrivial degrees, each with multiplicity at least 2, so $S$ is not a $T_{k}$-group.

(4) Assume that $S$ is a simple classical group in characteristic $p$.

$\left(4_{1}\right)$ Assume first that $S \notin \mathscr{L}$. We consider the following setup. Let $\mathscr{G}$ be a simply 
connected simple algebraic group of classical type and let $F$ be a suitable Frobenius map such that $L / Z(L) \cong S$, where $L=\mathscr{G}^{F}$. Let the pair $\left(\mathscr{G}^{*}, F^{*}\right)$ be dual to $(\mathscr{G}, F)$ and let $G=\left(\varphi^{*}\right)^{F^{*}}$. Let $T \leq G$ be a maximal torus of $G$. By Deligne-Lusztig theory, for each $G$-conjugacy class of regular semisimple element $s \in T$, there exists a semisimple character $\chi_{s} \in \operatorname{Irr}(L)$ with degree $|G: T|_{p^{\prime}}$ and if $s \in G^{\prime}$, then $\mathrm{Z}(L) \subseteq \operatorname{ker} \chi_{s}$, so $\chi_{s}$ is an irreducible character of $L / \mathrm{Z}(L) \cong S$. Moreover, if $t \in T \cap G^{\prime}$ is also a regular semisimple element which is not $G$-conjugate to $s$, then the semisimple character $\chi_{t} \in \operatorname{Irr}(L)$ is an irreducible character of $S$ with the same degree as that of $\chi_{s}$ and thus the nontrivial degree $|G: T|_{p^{\prime}} \in \operatorname{cd}(S)$ has multiplicity at least 2.

Since $S \notin \mathscr{L}$, by Lemma $3.1 G$ contains two maximal tori $T_{i}, i=1,2$, such that each $T_{i}^{\prime}=T_{i} \cap G^{\prime}$ possesses two regular semisimple elements $s_{i}$ and $t_{i}$ which are not $G$-conjugate. By the discussion above, we deduce that each nontrivial degree $\left|G: T_{i}\right|_{p^{\prime}} \in \operatorname{cd}(S)$ has multiplicity at least 2 . Since $\left|G: T_{i}\right|_{p^{\prime}}, i=1,2$, are distinct and nontrivial, we deduce that $S$ is not a $T_{k}$-group for any integer $k \geq 1$.

$\left(4_{2}\right)$ Assume next that $S \in \mathscr{L}$.

(a) Assume first that $S \cong \mathrm{PSL}_{2}(q)$ with $q \geq 4$. $\mathrm{As} \mathrm{PSL}_{2}(4) \cong \mathrm{PSL}_{2}(5) \cong \mathrm{A}_{5}$, we can assume that $q \geq 7$. If $q=7$, then $\mathrm{PSL}_{2}$ (7) is a $T_{2}$-group by using [Conway et al. 1985]. Hence we assume that $q \geq 8$. If $q$ is even, then $S \cong \operatorname{SL}_{2}(q)$ has degrees $q-1$ and $q+1$ with multiplicity $q / 2$ and $q / 2-1$, respectively. Since $q \geq 8$, we can see that $q / 2>q / 2-1 \geq 3$, so $S$ is not a $T_{k}$-group. Now assume that $q \geq 9$ is odd. Since $\mathrm{PSL}_{2}(9) \cong \mathrm{A}_{6}$, we can assume that $q \geq 11$. We know that $S$ has two irreducible characters of degree $(q+\epsilon) / 2$ where $q \equiv \epsilon(\bmod 4)$ and $\epsilon \in\{1,-1\}$. Furthermore, $S$ has a nontrivial degree $q-1$ with multiplicity $(q-\delta) / 4$, where $q \equiv \delta(\bmod 4)$ and $\delta \in\{1,3\}$. As $(q-1) / 4>(q-3) / 4 \geq(11-3) / 4=2$ and $q-1>(q+1) / 2 \geq(q-1) / 2$, $S$ has two distinct nontrivial degrees, each with multiplicity at least 2 and thus $S$ is not a $T_{k}$-group.

(b) Assume that $S \cong \operatorname{PSL}_{3}(q)$. Since $\mathrm{PSL}_{3}(2) \cong \mathrm{PSL}_{2}$ (7), we can assume that $q \geq 3$. For $3 \leq q \leq 11$, we can check that $\operatorname{PSL}_{3}(q)$ is not a $T_{k}$-group by using [Conway et al. 1985]. So, we assume that $q \geq 13$. In this case, by [Simpson and Frame 1973] $S$ has degrees $d_{1}=q^{2}+q+1$ and $d_{2}=q\left(q^{2}+q+1\right)$, both with multiplicity $(q-1) / d-1$. Since $q \geq 13$ and $d=\operatorname{gcd}(3, q-1) \leq 3$, it follows that $(q-1) / d-1 \geq(q-1) / 3-1 \geq 2$ and hence $S$ is not a $T_{k}$-group in these cases.

(c) Assume that $S \cong \operatorname{PSU}_{3}(q)$. Since $\mathrm{PSU}_{3}(2)$ is not simple, we can assume that $q \geq 3$. For $3 \leq q \leq 9$, we can check that $\operatorname{PSU}_{3}(q)$ is not a $T_{k}$-group by using [Conway et al. 1985]. So, we assume that $q \geq 11$. In this case, by [Simpson and Frame 1973] $S$ has degrees $d_{1}=q^{2}-q+1$ and $d_{2}=q\left(q^{2}-q+1\right)$, both 
with multiplicity $(q+1) / d-1$. Since $q \geq 11$ and $d=\operatorname{gcd}(3, q+1) \leq 3$, it follows that $(q+1) / d-1 \geq(q+1) / 3-1 \geq 2$. Thus $S$ is not a $T_{k}$-group.

(d) Assume that $S \cong \operatorname{PSp}_{4}(q)$ with $q \geq 3$. If $q \geq 4$ is even, then $S$ possesses two distinct nontrivial degrees $q\left(q^{2}+1\right) / 2$ and $(q-1)\left(q^{2}+1\right)$ with multiplicity 2 and $q$ respectively by using [Lübeck 2007]. Now assume that $q \geq 3$ is odd. Using [Lübeck 2007] again, $G$ has two distinct nontrivial character degrees $a_{1}:=2 q\left(q^{2}+1\right) / 2$ and $a_{2}:=(q+1)\left(q^{2}+1\right)$ with multiplicity 4 and $3(q-3) / 2$, respectively. Since $d=|G: S|=2$, we deduce that $a_{2} / d>a_{1}>d$. If $q \geq 5$, then $3(q-3) / 2 \geq 2 d=4$ and $4 \geq 2 d$, so it follows from Corollary 2.2 that $S$ is not a $T_{k}$-group. For the remaining cases, we can check directly using [Conway et al. 1985] that $S$ is not a $T_{k}$-group.

(e) Finally, for the remaining simple groups in $\mathscr{L}$, it is routine to check using [GAP 2012] that $S$ is not a $T_{k}$-group for any $k \geq 1$.

\section{Nonsolvable $T_{k}$-groups}

We first prove a special case of the main theorem. In fact, we show that nonperfect $T_{k}$-groups must be solvable. We note that if $G$ is a $T_{k}$-group for some integer $k \geq 1$ and $N \unlhd G$, then since $\operatorname{Irr}(G / N) \subseteq \operatorname{Irr}(G)$, we can easily see that $G / N$ is also a $T_{m}$-group for some integer $m \leq k$.

Theorem 4.1. If $G$ is a nonperfect $T_{k}$-group for some $k \geq 1$, then $G$ is solvable.

Proof. Let $G$ be a counterexample to the theorem with minimal order. Then $G^{\prime} \neq G$ and $G$ is a $T_{k}$-group for some $k \geq 1$ but $G$ is nonsolvable. Let $M$ be the last term of the derived series of $G$ and let $N \unlhd G$ such that $M / N$ is a chief factor of $G$. Since $G$ is nonsolvable, we see that $M$ is nontrivial and hence it is perfect, so $M / N$ is nonabelian and $M / N \cong W^{t}$ for some nonabelian simple group $W$ and some integer $t \geq 1$. Then $M / N$ is a minimal normal subgroup of $G / N$ and that

$$
\left|G / N:(G / N)^{\prime}\right|=\left|G / N: G^{\prime} / N\right|=\left|G: G^{\prime}\right|>1
$$

as $G$ is nonperfect and $N \leq M \leq G^{\prime}$. It follows that $G / N$ is a nonperfect nonsolvable group and since $\operatorname{Irr}(G / N) \subseteq \operatorname{Irr}(G)$, we deduce that $G / N$ is a nonperfect nonsolvable $T_{m}$-group for some integer $m \geq 1$. If $N$ is nontrivial, then $|G / N|<|G|$, which contradicts the minimality of $|G|$. Therefore, we conclude that $N$ must be trivial and $M \cong W^{t}$.

Claim 1. $M \cong \mathrm{PSL}_{2}\left(3^{f}\right)$ for some $f \geq 2$.

We first show that $W \cong \mathrm{PSL}_{2}\left(3^{f}\right)$ with $f \geq 2$. Suppose by contradiction that $W \varsubsetneqq$ $\operatorname{PSL}_{2}\left(3^{f}\right)$ with $f \geq 2$. Then there exist two irreducible characters $\theta_{i} \in \operatorname{Irr}(W)$ such that $\theta_{1}(1) \neq \theta_{2}(1)$ and both $\theta_{i}$ extend to $\operatorname{Aut}(W)$ by Lemma 2.3. Let $\varphi_{i}=\theta_{i}^{t} \in \operatorname{Irr}(M)$ for $i=1,2$. By [Bianchi et al. 2007, Lemma 5], we deduce that both $\varphi_{i}$ extend to 
$\chi_{i} \in \operatorname{Irr}(G)$. Furthermore, by Gallagher's theorem [Isaacs 1976, Corollary 6.17] we know that each $\varphi_{i}$ has exactly $\left|G / M:(G / M)^{\prime}\right|=\left|G: G^{\prime}\right|$ extensions. For each $i$, all extensions of $\varphi_{i}$ have the same degree which is $\varphi_{i}(1)=\theta_{i}^{t}(1)>1$. So, $G$ has two distinct nontrivial degrees $\theta_{i}^{t}(1), i=1,2$, both with nontrivial multiplicity, which is a contradiction. Hence $W \cong \mathrm{PSL}_{2}\left(3^{f}\right)$ with $f \geq 2$ as we wanted.

We now claim that $t=1$ and thus $M \cong \operatorname{PSL}_{2}\left(3^{f}\right)$ with $f \geq 2$. By way of contradiction, assume that $t \geq 2$. Let $\theta$ be the Steinberg character of $W$. Then $\varphi=\theta^{t} \in \operatorname{Irr}(M)$ extends to $\varphi_{0} \in \operatorname{Irr}(G)$ by [Bianchi et al. 2007, Lemma 5]. Thus by Gallagher's theorem [Isaacs 1976, Corollary 6.17] again, we have that $\varphi_{0}(1)=$ $\varphi(1)=\theta(1)^{t}$ is a nontrivial degree with nontrivial multiplicity. It follows that if $d \in \operatorname{cd}(G)$ with $1<d \neq \theta(1)^{t}=3^{t f}$, then the multiplicity of $d$ is trivial. It is well know that $\mathrm{PSL}_{2}\left(3^{f}\right)$ has two irreducible characters of degree $\left(3^{f}+\epsilon\right) / 2$, where $3^{f} \equiv \epsilon(\bmod 4)$ and $\epsilon \in\{ \pm 1\}$, hence $\mathrm{PSL}_{2}\left(3^{f}\right)$ has a nontrivial degree $\left(3^{f}+\epsilon\right) / 2<3^{f}$ with multiplicity 2 . Denote these two irreducible characters by $\alpha_{i}, i=1,2$. For $i=1,2$, let

$$
\varphi_{i}=1 \times 1 \times \cdots \times \alpha_{i} \in \operatorname{Irr}(M) \quad \text { and } \quad \psi=1 \times 1 \times \cdots \times \theta \in \operatorname{Irr}(M) .
$$

Let $I, I_{1}$ and $I_{2}$ be the inertia groups of $\psi, \varphi_{1}$ and $\varphi_{2}$, respectively. Obviously, we have that $M \leq I_{i} \leq I \leq G$ for $i=1,2$. By the representation theory of wreath products, we know that $\psi$ extends to $\psi_{0} \in \operatorname{Irr}(I)$ and $|G: I|=t$. Since $G / M$ is solvable, we deduce that $I / M$ is solvable. If $I / M$ is nontrivial, then $I / M$ has $j>1$ linear characters and thus $\psi$ has $j$ distinct extensions to $I$, which are $\psi_{0} \lambda$ with $\lambda \in \operatorname{Irr}(I / M)$ and $\lambda(1)=1$, so by Clifford's theorem [Isaacs 1976, Theorem 6.11] we have that $\left(\psi_{0} \lambda\right)^{G} \in \operatorname{Irr}(G)$ are distinct irreducible characters of $G$ having the same degree. Furthermore, for $\lambda \in \operatorname{Irr}(I / M)$ with $\lambda(1)=1$, we have

$$
\left(\psi_{0} \lambda\right)^{G}(1)=\psi_{0}^{G}(1)=|G: I| \psi_{0}(1)=3^{f} \cdot t<3^{t f} .
$$

The last inequality holds since $t \geq 2$. But then this is a contradiction since the multiplicity of the nontrivial degree $3^{f} \cdot t$ is at least $\left|I / M:(I / M)^{\prime}\right|$ which is nontrivial by our assumption. Therefore, we conclude that $I / M$ is trivial and so $I=M$. It follows that for $i=1,2$, we have $I_{i}=I=M$ since $M \leq I_{i} \leq I=M$. Thus for each $i$, we have $\varphi_{i}^{G} \in \operatorname{Irr}(G)$ and

$$
\varphi_{i}^{G}(1)=|G: M| \alpha_{i}(1)=|G: M|\left(3^{f}+\epsilon\right) / 2
$$

which is nontrivial and different from $3^{f t}$. Clearly, $\varphi_{1}^{G} \neq \varphi_{2}^{G}$, so we deduce that $G$ has a nontrivial degree $|G: M|\left(3^{f}+\epsilon\right) / 2 \neq 3^{f t}$ with multiplicity at least 2 , which is impossible. This contradiction proves our claim.

Claim 2. $G$ is an almost simple group with socle $M$.

By the previous claim, we know that $M \cong \mathrm{PSL}_{2}\left(3^{f}\right)$ with $f \geq 2$. Let $C=C_{G}(M)$. 
Then $C \unlhd G$ and $G / C$ is an almost simple group with socle $M C / C$. Assume first that $G / C$ is perfect. Then $G=M C$ and since $M$ is nonabelian simple, we must have that $M \cap C=1$ and so $G=M \times C$, where $G / M \cong C$ is solvable. If $C$ is nontrivial, then $\left|C: C^{\prime}\right|>1$ and so for each nontrivial irreducible character $\mu \in \operatorname{Irr}(M)$ of $M$, we see that $\mu$ has $\left|C: C^{\prime}\right|$ extensions to $G=M \times C$ and thus $G$ cannot be a $T_{k}$-group for any $k \geq 1$. Hence $C$ must be trivial and so $G$ is simple, which is impossible as $G \neq G^{\prime}$. Assume next that $G / C$ is nonperfect. Then $G / C$ is a nonperfect nonsolvable $T_{m}$-group for some $m \geq 1$. By the minimality of $|G|$, we must have that $C=1$ and thus $G$ is an almost simple group with socle $M \cong \mathrm{PSL}_{2}\left(3^{f}\right)$.

The final contradiction. Let $q=3^{f}$, with $f \geq 2$. Let $\alpha$ be an irreducible character of $M$ with $\alpha(1)=(q+\epsilon) / 2$ where $q \equiv \epsilon(\bmod 4)$ and $\epsilon \in\{ \pm 1\}$, let $\delta$ be the diagonal automorphism of $M$ and let $\varphi$ be the field automorphism of $M$ of order $f$. Then $\operatorname{Out}(M)=\langle\delta\rangle \times\langle\varphi\rangle$. Since $G$ is nonperfect, we deduce that $|G: M|$ is nontrivial. Observe that the Steinberg character $\mathrm{St}_{M}$ of $M$ is extendible to $\operatorname{Aut}(M)$ and so it extends to $G$ by [Bianchi et al. 2007, Lemma 5], hence by Gallagher's theorem [Isaacs 1976, Corollary 6.17] the degree $\mathrm{St}_{M}(1)=|M|_{3}$ has multiplicity at least $|G: M|>1$. Thus the multiplicity of every nontrivial degree of $G$ different from $|M|_{3}=q$ must be trivial. By [White 2013, Lemma 4.6], $\alpha$ is $\varphi$-invariant. Now if $G \leq M\langle\varphi\rangle$, then $\alpha$ is $G$-invariant and since $G / M$ is cyclic, $\alpha$ extends to $G$ and so $G$ has a nontrivial degree $(q+\epsilon) / 2$ with multiplicity at least $|G: M| \geq 2$, which is impossible. Hence $G \not \leq M\langle\varphi\rangle$. By [White 2013, Theorem 6.5], we have $I_{G}(\alpha)=G \cap M\langle\varphi\rangle$ and $\left|G: I_{G}(\alpha)\right|=2$. If $\left|I_{G}(\alpha): M\right|>1$, then $\alpha$ has $\left|I_{G}(\alpha): M\right|$ extensions to $I_{G}(\alpha)$ as $I_{G}(\alpha) / M$ is cyclic and thus by inducing these characters to $G$, we see that $G$ has at least $\left|I_{G}(\alpha): M\right| \geq 2$ irreducible characters of degree $q+\epsilon$, which is a contradiction. Thus, we conclude that $I_{G}(\alpha)=M$ and $|G: M|=2$. By [White 2013, Corollary 6.2], we have that either $G \cong \mathrm{PGL}_{2}(q)$ or $G \cong M\left\langle\delta \varphi^{f / 2}\right\rangle$, where $f$ is even. Clearly, the first case cannot happen. For the latter case, since $f$ is even, we obtain that $q \equiv 1(\bmod 4)$, so $M$ has exactly $(q-1) / 4$ irreducible characters of degree $q-1$. As $|G: M|=2$, by [White 2013, Theorem 6.6] all irreducible characters of $G$ lying over an irreducible character of $M$ of degree $q-1$ have degree $2(q-1)$. Therefore, $G$ has at least $(q-1) / 8$ irreducible characters of degree 2( $q-1)$. If $q=9$, then we can check directly using [Conway et al. 1985] that all almost simple groups with socle $\mathrm{PSL}_{2}(9) \cong \mathrm{A}_{6}$ are not $T_{k}$-groups for any integer $k \geq 1$. Thus we can assume that $q \geq 81$ as $f$ is even, so $(q-1) / 8 \geq 10$, hence $G$ is not a $T_{k}$-group. This final contradiction proves our theorem.

We are now ready to prove the main theorem.

Proof of Theorem A. Let $G$ be a counterexample to the theorem with minimal order. Then $G$ is a nonsolvable $T_{k}$-group for some integer $k \geq 1$ but $G$ is isomorphic 
to neither $\mathrm{PSL}_{2}(5)$ nor $\mathrm{PSL}_{2}$ (7). If $G^{\prime} \neq G$, then $G$ is solvable by Theorem 4.1, which is a contradiction. Thus we can assume that $G$ is perfect. Let $M$ be a maximal normal subgroup of $G$. Then $G / M$ is a nonabelian simple group. Since $\operatorname{Irr}(G / M) \subseteq \operatorname{Irr}(G)$, we deduce that $G / M$ is a $T_{m}$-group for some $m \geq 1$. Now by Theorem 3.2, we have that $G / M \cong \operatorname{PSL}_{2}(q)$ with $q \in\{5,7\}$ and $G / M$ is a $T_{2}$-group. Since $G$ is a counterexample to the theorem, we deduce that $M$ is nontrivial.

By [Conway et al. 1985], we know that $\operatorname{cd}\left(\mathrm{PSL}_{2}(5)\right)=\{1,3,4,5\}$ with multiplicity $1,2,1,1$ and $\operatorname{cd}\left(\mathrm{PSL}_{2}(7)\right)=\{1,3,6,7,8\}$ with multiplicity $1,2,1,1,1$. Since $G$ is a $T_{k}$-group, we deduce that the degree $3 \in \operatorname{cd}(G / M)$ is the unique nontrivial character degree in $\operatorname{cd}(G)$ with nontrivial multiplicity and also $k \geq 2$. If $k=2$, then $G$ must be isomorphic to either $\mathrm{PSL}_{2}(5)$ or $\mathrm{PSL}_{2}(7)$ by [Berkovich and Kazarin 1996, Main Theorem], which is a contradiction. Therefore, we must have that $k \geq 3$. Hence there exists $\chi \in \operatorname{Irr}(G \mid M)$ with $\chi(1)=3$ and thus $\chi \in \operatorname{Irr}(G \mid \theta)$ for some nontrivial irreducible character $\theta$ of $M$. By Clifford's theorem [Isaacs 1976, Theorem 6.2], we have that $\chi_{M}=e\left(\theta_{1}+\theta_{2}+\cdots+\theta_{t}\right)$, where all $\theta_{i}$ are conjugate to $\theta$ in $G, e \geq 1$ is the degree of an irreducible projective representation of $I_{G}(\theta) / M$ and $t=\left|G: I_{G}(\theta)\right|$. Since $\chi(1)=3$, we have that $3=\operatorname{et} \theta(1)$ and hence $t \leq 3$. As the index of a proper subgroup of $G / M$ with $G / M \cong \mathrm{PSL}_{2}(5)$ or $\mathrm{PSL}_{2}(7)$ is at least 5 , we must have that $t=1$, so $\chi_{M}=e \theta$ and $\theta$ is $G$-invariant. If $\theta$ is extendible to $\theta_{0} \in \operatorname{Irr}(G)$, then $\theta_{0}(1)=\theta(1) \geq 2$ since $G$ is perfect; also by Gallagher's theorem [Isaacs 1976, Corollary 6.17], we obtain that $\operatorname{Irr}(G \mid \theta)=\left\{\theta_{0} \lambda \mid \lambda \in \operatorname{Irr}(G / M)\right\}$. It follows that $\chi=\mu \theta_{0}$ for some $\mu \in \operatorname{Irr}(G / M)$. As $3=\chi(1)=\mu(1) \theta(1)$ and $\theta(1) \geq 2$, we must have that $\mu(1)=1$ and $\theta(1)=3$. Since $3 \in \operatorname{cd}(G / M)$ has multiplicity 2 , there exist two distinct irreducible characters $\lambda_{i}, i=1,2$, of $G / M$ with $\lambda_{i}(1)=3$ and so $\theta_{0} \lambda_{i}, i=1,2$, are two distinct irreducible characters of $G$, both have degree 9 . Thus $9 \in \operatorname{cd}(G)$ has multiplicity at least 2 which is a contradiction as $3 \in \operatorname{cd}(G)$ already has nontrivial multiplicity. Thus $\theta$ is $G$-invariant but it is not extendible to $G$. As the Schur multiplier of $\operatorname{PSL}_{2}(q)$ with $q \in\{5,7\}$ is cyclic of order 2, by the theory of character triple isomorphism [Isaacs 1976,

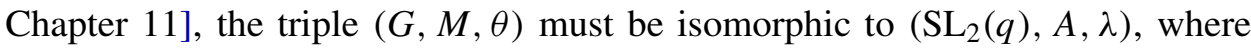
$q \in\{5,7\}, A=\mathrm{Z}\left(\mathrm{SL}_{2}(q)\right)$ and $\mu$ is a nontrivial irreducible character of $A$. Since $\operatorname{cd}\left(\mathrm{SL}_{2}(q) \mid \lambda\right)=\{(q-\epsilon) / 2, q-1, q+1\}$, with $q \equiv \epsilon(\bmod 4)$ and $\epsilon \in\{ \pm 1\}$, we deduce that $\operatorname{cd}(G \mid \theta)=\{\theta(1)(q-\epsilon) / 2,(q-1) \theta(1),(q+1) \theta(1)\}$. However, we can check that all degrees in $\operatorname{cd}(G \mid \theta)$ are even and thus $3 \notin \operatorname{cd}(G \mid \theta)$. This contradiction proves the theorem.

\section{Acknowledgments}

The author is indebted to G. Malle for his help with the proof of Lemma 3.1. The author is also grateful to the anonymous referee for useful comments and suggestions. 


\section{References}

[Babai et al. 2009] L. Babai, P. P. Pálfy, and J. Saxl, "On the number of p-regular elements in finite simple groups”, LMS J. Comput. Math. 12 (2009), 82-119. MR 2010f:20017 Zbl 1225.20013

[Berkovich 1996] Y. Berkovich, "Finite solvable groups in which only two nonlinear irreducible characters have equal degrees", J. Algebra 184:2 (1996), 584-603. MR 97j:20005b Zbl 0861.20008

[Berkovich and Kazarin 1996] Y. Berkovich and L. Kazarin, "Finite nonsolvable groups in which only two nonlinear irreducible characters have equal degrees", J. Algebra 184:2 (1996), 538-560. MR 97j:20005a Zbl 0861.20009

[Berkovich et al. 1992] Y. Berkovich, D. Chillag, and M. Herzog, "Finite groups in which the degrees of the nonlinear irreducible characters are distinct", Proc. Amer. Math. Soc. 115:4 (1992), 955-959. MR 92j:20006 Zbl 0822.20004

[Bianchi et al. 2007] M. Bianchi, D. Chillag, M. L. Lewis, and E. Pacifici, "Character degree graphs that are complete graphs", Proc. Amer. Math. Soc. 135:3 (2007), 671-676. MR 2007i:20014 Zbl 1112.20006

[Brauer 1963] R. Brauer, "Representations of finite groups", pp. 133-175 in Lectures on modern mathematics, I, edited by T. L. Saaty, Wiley, New York, 1963. MR 31 \#2314 Zbl 0124.26504

[Carter 1985] R. W. Carter, Finite groups of Lie type: Conjugacy classes and complex characters, Pure and Applied Mathematics 5, Wiley, New York, 1985. MR 87d:20060 Zbl 0567.20023

[Conway et al. 1985] J. H. Conway, R. T. Curtis, S. P. Norton, R. A. Parker, and R. A. Wilson, Atlas of finite groups, Oxford University Press, Eynsham, 1985. MR 88g:20025 Zbl 0568.20001

[Craven 2008] D. A. Craven, "Symmetric group character degrees and hook numbers", Proc. Lond. Math. Soc. (3) 96:1 (2008), 26-50. MR 2009f:20011 Zbl 1165.20008

[Deriziotis and Michler 1987] D. I. Deriziotis and G. O. Michler, "Character table and blocks of finite simple triality groups ${ }^{3} D_{4}(q)$ ”, Trans. Amer. Math. Soc. 303:1 (1987), 39-70. MR 88j:20011 Zbl 0628.20014

[Dolfi et al. 2013] S. Dolfi, G. Navarro, and P. H. Tiep, "Finite groups whose same degree characters are Galois conjugate", Israel J. Math. 198:1 (2013), 283-331. MR 3096641

[GAP 2012] The GAP Group, GAP - groups, algorithms, and programming, version 4.5.6, 2012, http://www.gap-system.org.

[Isaacs 1976] I. M. Isaacs, Character theory of finite groups, Pure and Applied Mathematics 69, Academic Press, New York, 1976. Reprinted Chelsea RI, 2006. MR 57 \#417 Zbl 0337.20005

[Larsen et al. 2013] M. Larsen, G. Malle, and P. H. Tiep, "The largest irreducible representations of simple groups”, Proc. London Math. Soc. 106:1 (2013), 65-96. Zbl 06139422

[Lübeck 2007] F. Lübeck, "Character degrees and their multiplicities for some groups of Lie type rank < 9", Online data, 2007, http://www.math.rwth-aachen.de/ Frank.Luebeck/chev/DegMult/ index.html.

[Malle 1999] G. Malle, "Almost irreducible tensor squares", Comm. Algebra 27:3 (1999), 1033-1051. MR 99m:20007 Zbl 0931.20009

[Malle 2008] G. Malle, "Extensions of unipotent characters and the inductive McKay condition", $J$. Algebra 320:7 (2008), 2963-2980. MR 2009e:20028 Zbl 1163.20003

[Malle 2010] G. Malle, "Zeros of Brauer characters and the defect zero graph", J. Group Theory 13:2 (2010), 171-187. MR 2011c:20016 Zbl 1208.20009

[Moretó 2007] A. Moretó, "Complex group algebras of finite groups: Brauer's problem 1", Adv. Math. 208:1 (2007), 236-248. MR 2008c:20011 Zbl 1109.20008 
[Seitz 1968] G. Seitz, "Finite groups having only one irreducible representation of degree greater than one", Proc. Amer. Math. Soc. 19 (1968), 459-461. MR 36 \#5212 Zbl 0244.20010

[Simpson and Frame 1973] W. A. Simpson and J. S. Frame, "The character tables for SL(3, q), $\mathrm{SU}\left(3, q^{2}\right), \operatorname{PSL}(3, q)$, PSU(3, $\left.q^{2}\right)$ ", Canad. J. Math. 25 (1973), 486-494. MR 49 \#398 Zbl 0264. 20010

[Suzuki 1962] M. Suzuki, "On a class of doubly transitive groups", Ann. of Math. (2) 75 (1962), 105-145. MR 25 \#112 Zbl 0106.24702

[Tong-Viet 2012] H. P. Tong-Viet, "Simple classical groups of Lie type are determined by their character degrees”, J. Algebra 357 (2012), 61-68. MR 2905242 Zbl 06104526

[Tong-Viet 2013] H. P. Tong-Viet, "Characterization of some simple groups by the multiplicity pattern”, Monatsh. Math. 172:2 (2013), 189-206. MR 3117188

[White 2013] D. L. White, "Character degrees of extensions of $\operatorname{PSL}_{2}(q)$ and $\operatorname{SL}_{2}(q)$,", J. Group Theory 16:1 (2013), 1-33.

[Zsigmondy 1892] K. Zsigmondy, "Zur Theorie der Potenzreste”, Monatsh. Math. Phys. 3:1 (1892), 265-284. MR 1546236 JFM 24.0176.02

Received October 25, 2012. Revised December 5, 2012.

HUNG P. TONG-VIET

School of Mathematics, Statistics and Computer Science

UNIVERSITY OF KWAZULU-NATAL

PRIVATE BAG X01

SCOTTSVILLE, 3209

SOUTH AFRICA

Current address:

FAKULTÄT FÜr MATHEMATIK

UNIVERSITÄT BIELEFELD POSTFACH 100131

D-33501 BIELEFELD

GERMANY

ptongviet@math.uni-bielefeld.de 


\title{
PACIFIC JOURNAL OF MATHEMATICS
}

\author{
msp.org/pjm
}

Founded in 1951 by E. F. Beckenbach (1906-1982) and F. Wolf (1904-1989)

\section{EDITORS}

Don Blasius (Managing Editor)

Department of Mathematics

University of California

Los Angeles, CA 90095-1555

blasius@math.ucla.edu

\author{
Paul Balmer \\ Department of Mathematics \\ University of California \\ Los Angeles, CA 90095-1555 \\ balmer@math.ucla.edu \\ Robert Finn \\ Department of Mathematics \\ Stanford University \\ Stanford, CA 94305-2125 \\ finn@math.stanford.edu \\ Sorin Popa \\ Department of Mathematics \\ University of California \\ Los Angeles, CA 90095-1555 \\ popa@math.ucla.edu
}

\author{
Vyjayanthi Chari \\ Department of Mathematics \\ University of California \\ Riverside, CA 92521-0135 \\ chari@math.ucr.edu \\ Kefeng Liu \\ Department of Mathematics \\ University of California \\ Los Angeles, CA 90095-1555 \\ liu@math.ucla.edu \\ Jie Qing \\ Department of Mathematics \\ University of California \\ Santa Cruz, CA 95064 \\ qing@ cats.ucsc.edu
}

\section{PRODUCTION}

Silvio Levy, Scientific Editor, production@msp.org

\section{SUPPORTING INSTITUTIONS}

ACADEMIA SINICA, TAIPEI

CALIFORNIA INST. OF TECHNOLOGY

INST. DE MATEMÁTICA PURA E APLICADA

KEIO UNIVERSITY

MATH. SCIENCES RESEARCH INSTITUTE

NEW MEXICO STATE UNIV.

OREGON STATE UNIV.

\author{
STANFORD UNIVERSITY \\ UNIV. OF BRITISH COLUMBIA \\ UNIV. OF CALIFORNIA, BERKELEY \\ UNIV. OF CALIFORNIA, DAVIS \\ UNIV. OF CALIFORNIA, LOS ANGELES \\ UNIV. OF CALIFORNIA, RIVERSIDE \\ UNIV. OF CALIFORNIA, SAN DIEGO \\ UNIV. OF CALIF., SANTA BARBARA
}

\author{
Daryl Cooper \\ Department of Mathematics \\ University of California \\ Santa Barbara, CA 93106-3080 \\ cooper@math.ucsb.edu \\ Jiang-Hua Lu \\ Department of Mathematics \\ The University of Hong Kong \\ Pokfulam Rd., Hong Kong \\ jhlu@maths.hku.hk \\ Paul Yang \\ Department of Mathematics \\ Princeton University \\ Princeton NJ 08544-1000 \\ yang@math.princeton.edu
}

These supporting institutions contribute to the cost of publication of this Journal, but they are not owners or publishers and have no responsibility for its contents or policies.

See inside back cover or msp.org/pjm for submission instructions.

The subscription price for 2014 is US $\$ 410 /$ year for the electronic version, and \$535/year for print and electronic.

Subscriptions, requests for back issues and changes of subscribers address should be sent to Pacific Journal of Mathematics, P.O. Box 4163, Berkeley, CA 94704-0163, U.S.A. The Pacific Journal of Mathematics is indexed by Mathematical Reviews, Zentralblatt MATH, PASCAL CNRS Index, Referativnyi Zhurnal, Current Mathematical Publications and Web of Knowledge (Science Citation Index).

The Pacific Journal of Mathematics (ISSN 0030-8730) at the University of California, c/o Department of Mathematics, 798 Evans Hall \#3840, Berkeley, CA 94720-3840, is published twelve times a year. Periodical rate postage paid at Berkeley, CA 94704, and additional mailing offices. POSTMASTER: send address changes to Pacific Journal of Mathematics, P.O. Box 4163, Berkeley, CA 94704-0163.

PJM peer review and production are managed by EditFLOW ${ }^{\circledR}$ from Mathematical Sciences Publishers.

\section{PUBLISHED BY}

\section{mathematical sciences publishers \\ nonprofit scientific publishing}

http://msp.org/

(C) 2014 Mathematical Sciences Publishers 


\title{
PACIFIC JOURNAL OF MATHEMATICS
}

\author{
Volume $268 \quad$ No. $2 \quad$ April 2014
}

In memoriam: Jonathan Rogawski

DON BLASIUS, DINAKAR RAMAKRISHNAN and V. S. VARADARAJAN

Formes modulaires sur la $\mathbb{Z}_{p}$-extension cyclotomique de $\mathbb{Q}$

LAURENT CLOZEL

Weight zero Eisenstein cohomology of Shimura varieties via Berkovich

275 spaces

MICHAEL HARRIS

$\Lambda$-adic Barsotti-Tate groups

HARUZO HIDA

Le flot géodésique des quotients géométriquement finis des géométries de

Hilbert

MickaËl CRAMPON and LUdOViC MARQuis

Nonplanarity of unit graphs and classification of the toroidal ones

A. K. Das, H. R. Maimani, M. R. Pournaki and S. Yassemi

Discrete semiclassical orthogonal polynomials of class one

DIEGO DOMINICI and FRANCISCO MARCELLÁN

A note on conformal Ricci flow

PENG LU, Jie QING and Yu Zheng

On representations of $\mathrm{GL}_{2 n}(F)$ with a symplectic period

ARNAB Mitra

Linked triples of quaternion algebras

ALEXANDER S. SIVATSKI

Finite nonsolvable groups with many distinct character degrees

Hung P. TONG-VIET

Errata to "Dynamics of asymptotically hyperbolic manifolds"

JULIE ROWLETT

Erratum to "Singularities of the projective dual variety" 